\title{
DETERMINATION OF CUTTING FORCES IN GRINDING WITH CROSSED AXES OF TOOL AND WORKPIECE
}

\author{
Volodimyr KALCHENKO*, Andrij YEROSHENKO*, Sergiy BOYKO*, Nataliia SIRA* \\ "Chernihiv National University of Technology, Mechanical Engineering Department, 95 Shevchenko street, Chernihiv, Ukraine \\ vvkalchenko74@gmail.com, yeroshenkoam@gmail.com, svboyko.cstu@gmail.com, nnseraya@ukr.net
}

received 6 May 2016, revised 10 March 2017, accepted 13 March 2017

\begin{abstract}
In the work the analysis of existent methods of determination of local and general forces of cutting at polishing of surfaces with a type as the arc of circumference is given. The dependence for determination of speed polishing and method for determination of thickness of the cut away layer on condition of equality of the tricked into and taken off volumes of material are offered. The method of determination of cutting forces, which takes into account cutting and deforming grain, is suggested. The method of determining the thickness of a cutting layer of one of the cutting edge, from the condition that the volume of material that is brought and is cut in each local point of contact spots has been proposed. The proposed method takes into account the compliance of the processing system and the discontinuity of the abrasive surface of the tool. By experimental way upper limits of thickness cutting layer when using different abrasive materials for a wide range of cutting speeds have been obtained.
\end{abstract}

Keywords: Cutting Forces, Grinding, Deforming Grain, Cutting Edge, Abrasive Surface, Abrasive Materials, Crossed Axes, Abrasive Grain, Cut Volumes, Grinding Wheel

\section{INTRODUCTION}

Application in manufacturing of the new methods of grinding requires the development of more accurate methods for calculating of cutting forces. There are two methods of determining the constituents of cutting forces: an empirical and calculatedexperimental. The first method is based on measuring the cutting force by dynamometers, when grinding samples with the following obtaining empirical relationships. However, empirical relationships can only be used for calculations in the conditions close to the experimental, that limits their use. Calculationexperimental method defines a single force acting on the one cutting edge. After that the number of cutting edges, which are located in the contact zone of the abrasive wheel with the workpiece are determined, and then the resulting cutting force is calculated (Chang et al., 2008; Hecker, Rogelio, 2007).

Existing methods of determining of cutting forces (Malkin and Guo, 2008; Yeroshenko, 2009) do not take into account the influence of deforming grains, the oscillation power in the local areas of the contact patch, the rigidity of the processing system and intermittent surface of the wheel. The calculated volume of material that is removed by the cutting edge does not conform the volume which is fed to the cutting zone(Yeroshenko, 2009; Chen et al., 2007; Chee Keong Ng, 2006). This is explained by the fact that when determining of cutting forces the average value of a cut off volume is calculated and thus a material that is removed at the local site of the contact patch is not considered.

Thus, the aim of the work is the determination of local and total cutting forces based on the equality of supplied and cutting volumes, taking into account static compliances of processing systems and discontinuous of the tool surfaces.

\section{DETERMINATION OF THE CUTTING LAYER THICKNESS}

For reaching of the set goal, it is necessary to determine the layer thickness $\mathrm{a}_{\mathrm{z}}$, which is cut by one edge. The equation for determining the thickness of a cut off layer is the fundamental equation of grinding. The layer thickness determines the load on the grain, resistance and maximum cutting ability of the abrasive tool, the wear of the abrasive grains, the quality and depth of scratches on the workpiece. By experimental way upper limits azmax of thickness cutting layer when using different abrasive materials for a wide range of cutting speeds have been obtained. The slice thickness and their shape are determined by the kinematics of the process, the number and shape of the edges, that take part in the work of grinding (Kalchenko and Yeroshenko, 2009; Yeroshenko, 2008).

The main kinematic indicators that characterizes the process of cutting in the local point of contact patch is the ratio of $V_{n} / V_{s}$, where $V_{n}=\vec{V} \cdot \vec{n}$ - the projection of the relative speed of $\vec{V}$ on the normal $\overrightarrow{\mathrm{n}}$ to the surface of the tool; $V_{g}$ - grinding speed (the projection of relative speed $\vec{V}$ on the surface of instrument).

It must be noted that, while processing of parts with a profile in the form of a circular arc, the grinding speed along generatrix of profile is variable. The oscillation of speed along the profile becomes significant with a reduction ratio $R_{t} / \rho_{t}$ (Fig. 1). Based on Fig. 1, the rate of grinding in the j-th point can be determined by the formula:

$V_{g j}=\frac{2 \pi\left(R_{t}+\rho_{t} \cdot \cos \varphi_{t j}\right) \cdot n}{1000}$

where: $n$ - rotational speed of wheel; $R_{t}$ - radius of the center of the circle profile; $\rho_{t}$ - the radius of the circle profile; $\varphi_{t j}$ - the 
angular coordinate of the tool.

There are two approaches to the definition of cutting layer thickness:

- a kinematic approach considers the cut geometry in the plane normal to the cutting surface (Yeroshenko, 2009; Stepien, 2007), asking the average distance between the cutting edges;

- a volumetric approach involves determining of the average volume of the slice and taking its shape and the average number of cutting edges on one surface of the wheel, finding its thickness (Yeroshenko, 2009; Kalchenko et al., 2012).

When calculating the cutting layer thickness it is necessary to provide cutting of all material that is fed into the processing zone. Since the location of the grain on the tool surface is chaotic, and they have a different shape, you must use a volume approach. The shape of the cutting edge (Fig. 2) has the shape of a cone with an apex angle $\alpha=90-160^{\circ}$ and a radius of curvature $\rho$ (Yeroshenko, 2009; Chae et al., 2006).

The slice area equals the cross sectional area of abrasive grains and depends on the thickness of slice $\mathrm{a}_{\mathrm{z}}$, the radius of curvature $\rho$ of the cutting edge and the selected variant of the slice form (Yeroshenko, 2009; Chae et al., 2006). Fig. 3 shows this dependence for $\rho=0$ (curve 1) and $\rho=80$ microns (curve 2).

At scoping, which is cut off by a cutting blade it is necessary to take into account the stiffness of the treated system, since the elastic deformation of the system machine-detail lead to additional errors. The stiffness of the treated system can be taken into account through pliability $y_{s}=f\left(\varphi_{t j}, K_{s}\right)$, which is a function of the angular position of a point on the profile $\varphi$ and coefficient static compliance $K_{s}$.

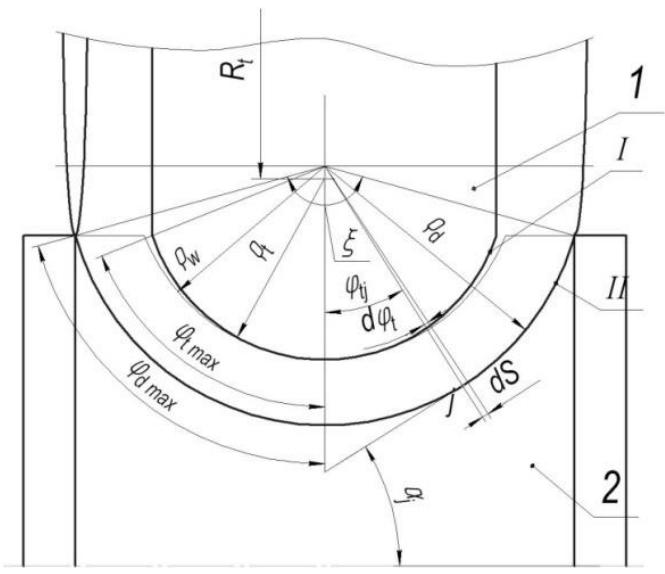

Fig. 1. Processing with crossed axes tool and workpiece with a profile in the form of a circular arc $\left(\rho_{d}\right.$ - radius of the detail profile; $\xi$ - the central angle of the detail profile; $\rho_{w}$ - radius of the workpiece profile; $\varphi_{\text {tmax }}$ - the max angular coordinate of the tool; $\varphi_{\text {dmax }}$ - the max angular coordinate of the detail; $\alpha_{j}-$ tilt angle of the tangent to the circle surface at the point $j$; $d_{\varphi t}$ - angular coordinate increment; 1 - wheel; 2 - detail; I - starting position of the wheel; II - final position of the wheel)

In passing from one cutting edge to the elementary portion of wheel $d S$ one must considere the nonuniformity surface of an abrasive tool. It is possible to take into account the discontinuity surface of the wheel, by the usage of coefficient: $\left[1-\exp \left(-\frac{\Sigma \mathrm{b}(\mathrm{t}, \theta)}{\mathrm{b}_{0}}\right)\right]$ (Yeroshenko, 2009).

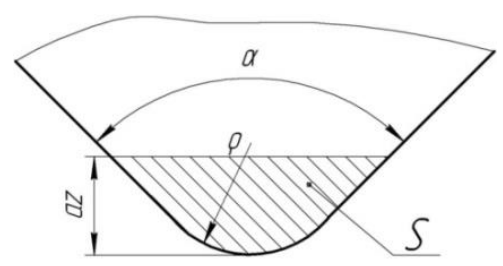

Fig. 2. The shape of the abrasive grain

Thus, in the grinding on the elementary area dS (Fig. 4) per unit time it is fed $\left(V_{n}-y_{s}\right)-\left[1-\exp \left(-\frac{\Sigma b(t, \theta)}{b_{0}}\right)\right] d S$ of the material. During the same time dSarea, the surface of which has $N_{z}\left(a_{z}\right)$ cutting edges, removes $N_{z}\left(a_{z}\right) \cdot V_{s}\left(\varphi_{t j}\right) \cdot S\left(a_{z}, \rho\right)$ of material. Based on the condition of the equality of the removed and cut volumes, you can create an expression:

$$
\begin{aligned}
\left(V_{n}-y_{s}\right) \cdot\left[1-\exp \left(-\frac{\Sigma b(t, \theta)}{b_{0}}\right)\right] \cdot d S \\
=N_{z}\left(a_{z}\right) \cdot V_{S}\left(\phi_{t j}\right) \cdot S\left(a_{z}, \rho\right)
\end{aligned}
$$

where: $N_{z}\left(a_{z}\right)$ - number of cutting edges on a given level $\mathrm{a}_{\mathrm{z}}$ and disposed on the unit area of a wheel, which participate in the cutting of metal; $S\left(a_{z}, \rho\right)$ - sectional area of the abrasive grain. From the expression (2) it is determined the generalized equation of the shear layer:

$$
\frac{\left(V_{n}-y_{S}\right)}{V_{S}\left(\phi_{t j}\right)}=\frac{N_{z}\left(a_{z}\right) \cdot S\left(a_{z}, \rho\right)}{\left[1-\exp \left(-\frac{\Sigma b(t, \theta)}{b_{0}}\right)\right] \cdot d S} .
$$

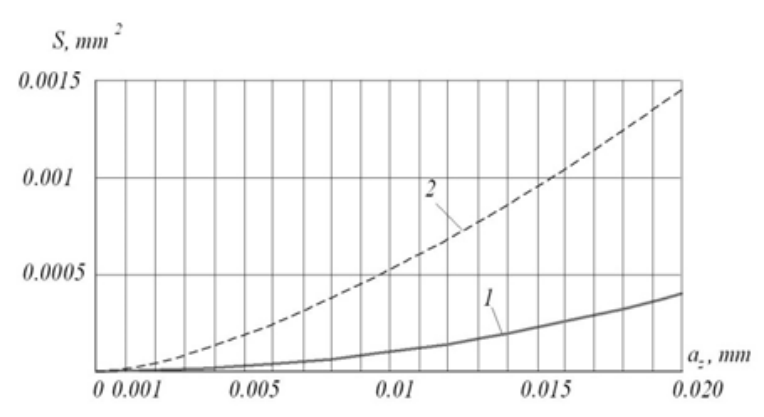

Fig. 3. The dependence of a cut off layer area from the slice thickness and the radius of edge curvature

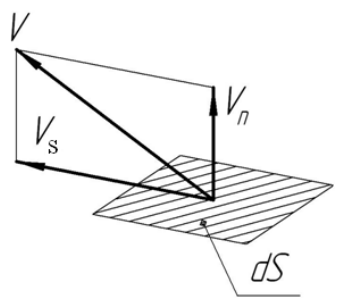

Fig. 4. The scheme for determining the thickness of a cut off layer

The abrasive grain, which is located on the working surface of the wheel, it is accepted to characterize by a number of parameters. Thus, according to modern concepts (Heinzel and Bleil, 2007;Yan and Lan, 2009), it is necessary to specify:

- The location of the top level of the grain regarding the level of the most protruding grains;

- Corner radius grain $\rho$. 
The density of the probability location of the cutting edge $\mathrm{fN}(\mathrm{x})$, at the level $\mathrm{z}$ from the outer surface of the wheel, can be described by the law beta distribution (Yeroshenko, 2009):

$f_{N}(x)=A \cdot t^{\gamma-1} \cdot(1-t)^{\eta-1}$

where: $x=\frac{z}{h_{n}}$ - equivalent level of the grinding wheel, on which it is determined $f_{N}(x) ; A, n, \gamma, \eta$ - the parameters of the beta distribution; $h_{n}$ - the total height of the profile of the grinding wheel.

The total number of cutting edges on level $x$ can be determined (Yeroshenko, 2009; 2008):

$N_{z}(x)=N_{n} \cdot A \cdot \int_{0}^{z / h_{n}} t^{\gamma-1}(1-t)^{\eta-1} \mathrm{dt}$,

where: $N_{z}(x)$ - the number of cutting edges on a unit area on the level $x ; N_{n}$ - total number of cutting edges along the entire height $h_{n}$ profile on the unit area.

Similarly we define the probability density of the radii distribution of the cutting edges curvature $\rho$ :

$f_{\rho}(x)=A \cdot t^{\gamma-1} \cdot(1-t)^{\eta-1}$

where: $x=\rho / \rho_{\max }$ - relative radius of curvature of the cutting edge; $\rho_{\max }$ - the maximum radius of the cutting edge.

Dependence 5 for grinding wheel AA-70-K7-V35 is shown in Fig. 5.

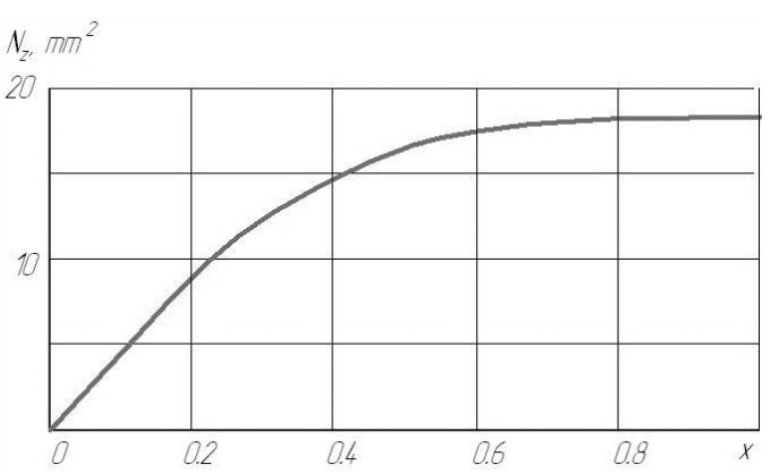

Fig. 5.The distribution of the number of cutting edges and fillet radii

However, not all cutting edges remove metal themselves. Some of them deform the metal elastically or plastically. It is considered (Ng and Chee Keong, 2006; Oliveira, 2009) that the criterion for cutting is the ratio $\mathrm{z} / \rho$. To determine the minimum value of this ratio it is used experimental dependence $(\mathrm{Ng}$ and Chee Keong, 2006; Oliveira, 2009), which describes the minimum thickness of the cut layer, depending on the radius of curvature of the top of the grain and the cutting speed. In this case the minimum value of the ratio the cutting process is still going on. By approximating the experimental dependence by the exponential function (Yeroshenko, 2009; 2008), you can record it in the general form:

$\frac{z}{\rho}=B \cdot \rho^{-\alpha} \cdot V_{s}^{-\beta}\left(\phi_{\mathrm{tj}}\right)$

where: $B, \alpha, \beta$ - empirical coefficients determined depending on the material, the material of tool and workpiece.

Taking into account (7), you can obtain the dependence that will serve as a lower limit of the determination of the probability of cutting grain at depth $z$ in the $j$-th point (Fig. 6):

$$
z=B \cdot \rho^{1-\alpha} \cdot V_{s}^{-\beta}\left(\phi_{\mathrm{tj}}\right) .
$$

As the grinding speed $V_{s}\left(\varphi_{t j}\right)$ is in a function at dependence of the angular position of point $j$, it will vary within the generatrix profile and thus it will change the depth $z$ in the range of $z_{\text {min }}$ to $z_{\max }$, which correspond $V_{s}\left(\varphi_{t}=0\right)$ and $V_{s}\left(\varphi_{\operatorname{tmax}}\right)$. Changing of the depth $z$ along generatrix length of a profile will lie within area bounded by two curves in Fig. 6 .

Statistical research of the work surface parameters (Yeroshenko, 2009; Nguyen and Butler, 2005; Brinksmeire, 2006) based on the density distribution found that the grains which deform metal in the cutting area is significantly greater than those that cut (area under the graph in Fig. 6).

Edges which are disposed below the metal level of penetration $\mathrm{z}$ in the grinding wheel, are involved in metal processing. Level $z$ depends on the attitude and takes different values for each point of the contact patch. That's why, this scheme is given for a specific point of a contact patch, but rather for a specific relationship $\frac{\left(V_{n}-y_{s}\right)}{V_{s}\left(\varphi_{t j}\right)}$. Lines $z\left(\rho, V_{s}\right)$ divide the edge of the abrasive grains to the cutting and deforming.

Graph analysis allows to state that while an increase of cutting speed the minimum thickness of cutting layer decreases. Since certain values of the depth $z$, the proportion of grains, which are involved in metal cutting, increases considerably. At shallow depths of cut the grain particle that is involved in cutting, should have only the smallest values of the radius of curvature of the cutting edge. After some time of operation, due to the occurrence of wear areas this share is shrinking.

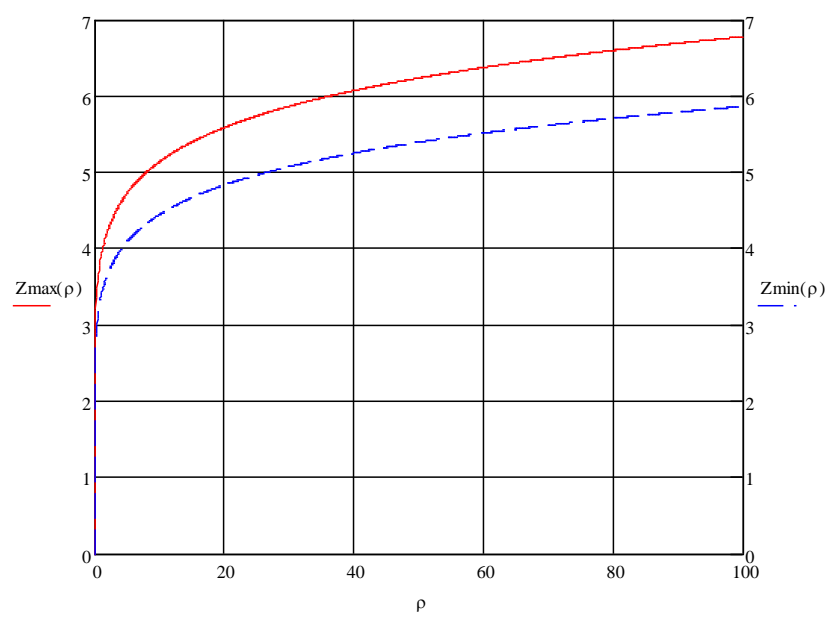

Fig. 6.Graph of the dependence of depth $z$, which defines the beginning of the cutting process by the edge of the abrasive tool

\section{THE NUMBER OF CUTTING AND DEFORMING EDGES}

The number of cutting and deforming edges which fall on the surface of the wheel unit can be determined by the following relationships (Yeroshenko, 2009):

$N_{z}^{p}=\iint_{S_{c}} N_{n} \cdot f_{N}(z) \cdot f_{\rho}(\rho) \cdot \mathrm{ds}$,
$N_{z}^{D}=\iint_{S_{d}} N_{n} \cdot f_{N}(z) \cdot f_{\rho}(\rho) \cdot \mathrm{ds}$,

where: $S_{c}$ and $S_{d}$ - the area in Fig. 7 which correspond to the cutting and deforming edges respectively. 
Removal of metal provides exactly cutting edge, so it is of their number will depend on the thickness ofa cut off layer. Therefore, the equation (3) must be written in the form:

$$
\frac{\left(V_{n}-y_{\mathrm{s}}\right)}{V_{S}\left(\phi_{\mathrm{tj}}\right)}=\frac{N_{z}^{p}\left(a_{z}\right) \cdot S\left(a_{z}, \rho\right)}{\left[1-\exp \left(-\frac{\Sigma b(t, \theta)}{b_{0}}\right)\right] \cdot \mathrm{d} S} .
$$

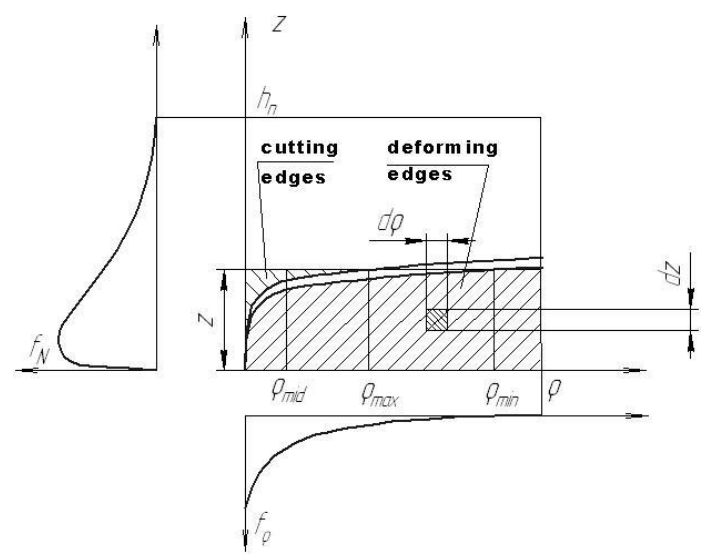

Fig. 7.Distribution of cutting and deforming edges in the contact patch

To determine the thickness of a cut off layer it is necessary to know the average radius of curvature of the grain edges, which take part in cutting at a given point in the contact patch. To simplify the calculations we assume:

$\rho_{\text {mid }}=\frac{\rho^{\prime}}{3}$

where: $\rho^{\prime}$ - the coordinate of the intersection of levels with a linelevel $z\left(\rho, V_{s}\right)$, or $\rho_{\max }$ when $\rho^{\prime}>\rho_{\max }$.

The dependence of a cut off layer thickness $\mathrm{a}_{\mathrm{z}}$ on the ratio $\frac{\left(V_{n}-y_{s}\right)}{V_{S}\left(\varphi_{t j}\right)}$ can be determined by joint decision of $4,6,8,9,11,12$ equations. The graph of this dependence is shown in Fig. 8.

$$
a_{z}, \mathrm{~mm}
$$

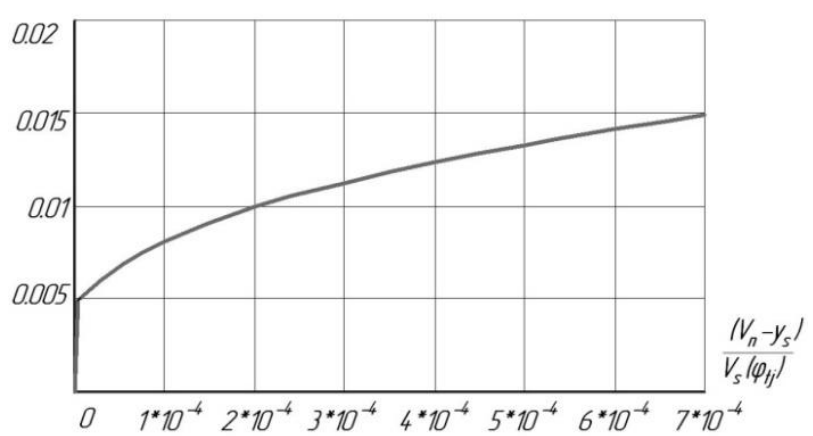

Fig. 8. The dependence of the thickness of a cut off layer $a_{z}$ on the ratio $\frac{\left(V_{n}-y_{s}\right)}{V_{s}\left(\varphi_{t j}\right)}$

Knowing the dependence $a z \frac{\left(V_{n}-y_{s}\right)}{V_{s}\left(\varphi_{t j}\right)}$ can obtain from the formulas (9) and (10) dependences $N_{z}^{c}\left(\frac{\left(V_{n}-y_{s}\right)}{V_{s}\left(\varphi_{t j}\right)}\right)$ and $N_{z}^{d}\left(\frac{\left(V_{n}-y_{s}\right)}{V_{s}\left(\varphi_{t j}\right)}\right)$, which graphs are shown in Fig. 9.

\section{DETERMITATION OF THE CUTTING FORCES}

It is considered (Yeroshenko, 2009) that the separate cutting edge is under action of the shear force $P_{s d}$, friction $P_{f}$ and inertia $P_{\text {in }}$. Dependence to determine the normal and tangential forces acting on the cutting edge, taking into consideration these components are given in work of Yeroshenko, 2009. Total cutting force is determined by integrating of the microcutting forces on the contact patch surface based on cutting and deforming grains.

From the foregoing, the total tangential $P_{z}^{\Sigma}$ and normal $P_{n}^{\Sigma}$ cutting force can be determined by dependencies:

$P_{z}^{\Sigma}=\iint_{S} P_{z}^{c} \cdot n_{c}(z, \rho) \cdot \mathrm{dS}+\iint_{S} P_{z}^{d} \cdot n_{d}(z, \rho) \cdot \mathrm{dS}$,

$P_{n}^{\Sigma}=\iint_{S} P_{n}^{c} \cdot n_{c}(z, \rho) \cdot \mathrm{d} \mathrm{S}+\iint_{S} P_{n}^{d} \cdot n_{d}(z, \rho) \cdot \mathrm{d} \mathrm{S}$

where: $P_{z}^{c}, P_{z}^{d}$ - forces which act in the tangential direction, respectively, on the cutting edge and deforming; $n_{c}, n_{d}$ - number, respectively, of cutting and deforming edges on the elementary area $d S ; P_{n}^{c}, P_{n}^{d}$ - forces that operate in the normal direction, respectively, on the cutting edge and deforming; $S$ - the spot of the contact area of the wheel and the parts.

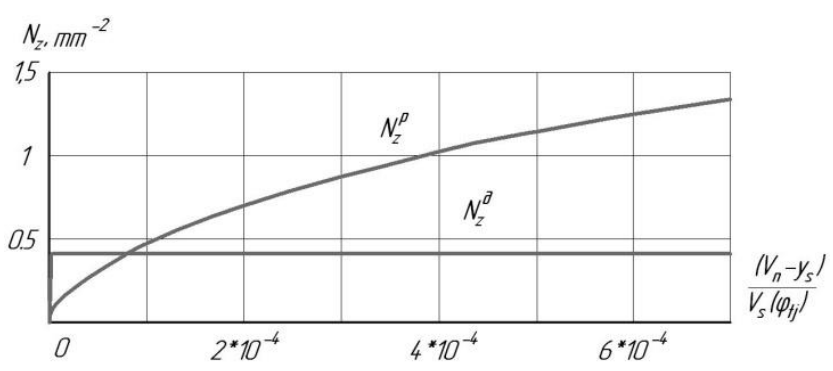

Fig. 9. The dependence of the number of cutting and deforming edges from the ratio $\frac{\left(V_{n}-y_{s}\right)}{V_{s}\left(\phi_{t i}\right)}$

The shape and size of the contact area depends on the surface treatment process with a profile in the form of a circular arc. The calculation of the contact line of a part and a grinding wheel carried out by the method described in work of Yeroshenko (2009). Spot contact is the region bounded by the contact line and the cross-section of the grinding wheel, shifted by the amount of feed, the workpiece. In the traditional infeed grinding (Yeroshenko, 2009), when the radius of the tool $\rho_{t}$ equals the profile radius of the machined workpiecepw and processing is done only with cross feed, the form of contact area will be almost a rectangle projected on the treated surface (Fig. 10). Contact area is bounded by a line contact 1 , along which the shaping surface of the workpiece is done and on which the length $V_{n}=$ 0 , and the output line of the grinding wheel 2 , a maximum value $\mathrm{V}_{\mathrm{n}}$. On each side the contact area is limited to links section of the grinding wheel with the workpiece with outer cylinder 3 , and 4 .

When processing with crossed axes tool and workpiece with a profile in the form of circular arcs machining is produced by a spiral of Archimedes. Permanent of Archimedean spiral, in which the relative motion moves the wheel with renting of allowance $\delta_{i s} a=\frac{t}{2 \pi}$. The line and the contact area of the spot with this method of processing have a spatial form (Fig. 10), and the radius of the line projection on the axial of the plane of contact during final shaping surface of a part is equal to the radius profile of a part. 


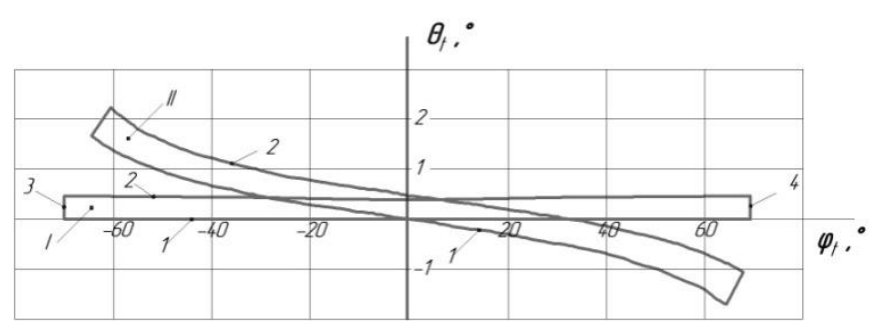

Fig. 10.The contact spots (I) with traditional and the new (II) methods of surface treatment with a profile in the form of a circular arc

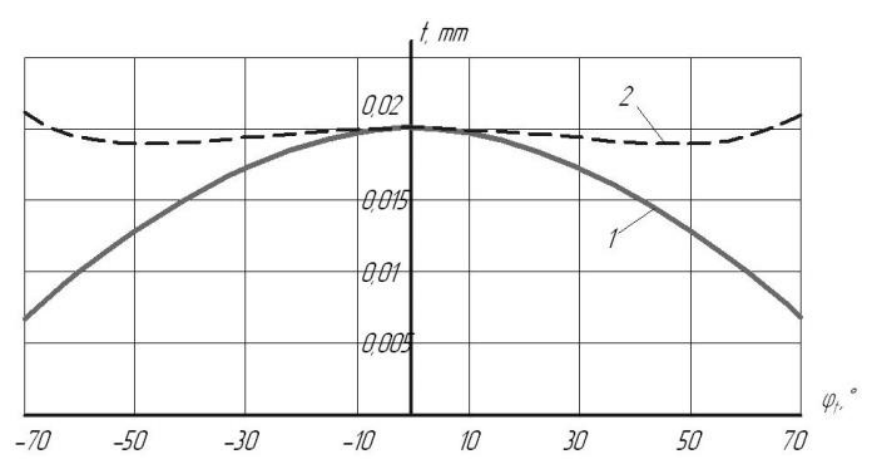

Fig. 11. Allocation of machining allowance that is cut off in one revolution of the workpiece, on the arc chute

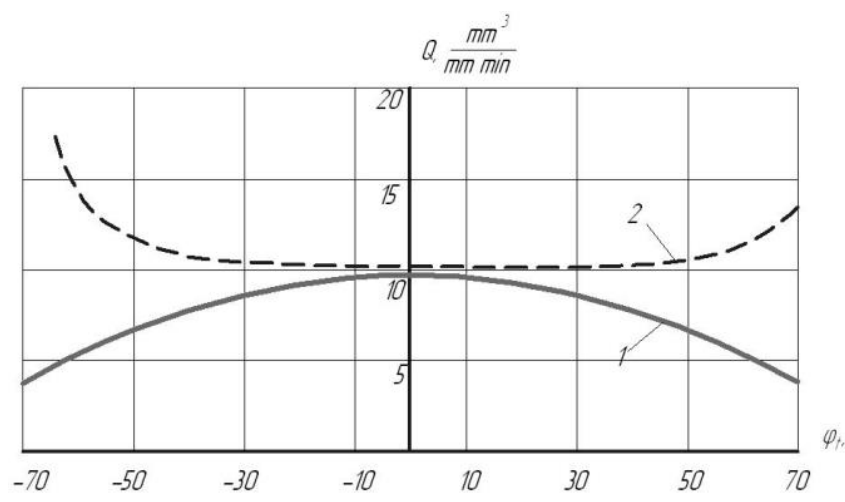

Fig. 12. Distribution of specific grinding performance on the arc chute

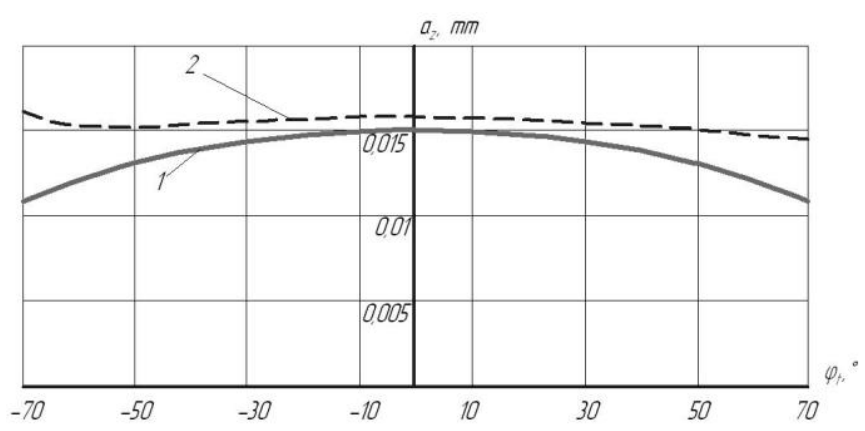

Fig. 13. Distribution of a cut off layer thickness by a cutting edge on the output line of the grinding wheel of the machining allowance

The allowance, cut in one revolution of the workpiece (Fig. 11 ) is defined as the distance between the workpiece and the part surface. Curve 1 illustrates the processing of the traditional way, curve 2 - processing with crossed axes of a tool and workpiece.

Immediate grinding performance that describes the volume of material which is removed by the contact line is defined by the expression:

$$
\begin{aligned}
& Q=\int_{-\phi_{t \max }}^{+\phi_{\operatorname{tmax}}}\left(\int_{\theta_{1 k j}}^{\theta_{2 k j}}\left(\bar{V}_{j} \bar{n}_{j}-y_{s}\right)\right. \\
& \cdot\left(R_{t}+\left(\rho_{t}-W_{j}\right) \cos \varphi_{t j}\right. \\
& \left.\left[1-\exp \left(-\frac{\Sigma b(t, \theta)}{b_{0}}\right)\right] \cdot d \theta_{k}\right) \\
& \cdot \sqrt{\left.\left(\frac{d R_{\varphi_{t j}}}{d j}\right)^{2}+\left(\frac{d \varphi_{t j}}{d j}\right)^{2}\right)} \cdot d \varphi_{t}
\end{aligned}
$$

where: $\pm \varphi_{\text {tmax }}$ - limiting value of angular position $\varphi_{t j}$ of point $j$ of the wheel profile; $\theta_{1 k j}, \theta_{2 k j}$ - angular coordinates of the contact patch on the radius $\left(R_{t}+\rho_{t} \cdot \cos \phi \varphi_{t j}\right)-W_{j}$. $\cos \varphi_{t j}=R_{t}+\left(\rho_{t}-W_{j}\right) \cdot \cos \varphi_{t j}-$ volumetric wear wheel at the point $j ; \bar{V}_{j}, \bar{n}_{j}$ - vectors of the cutting speed and the normal to the j-th point of the wheel; $y_{s}=f\left(\varphi_{t j}, K_{s}\right)$ - compliance of the processing system; $K_{s}$ - the value of the static compliance; $R_{t}$ - the distance from the axis of rotation of the wheel to the center of its profile $\rho_{t}$ radius; $R_{\varphi_{t j}}=R_{t}+\rho_{t} \cos \varphi_{t j}$ - radius of rotation j-th point.

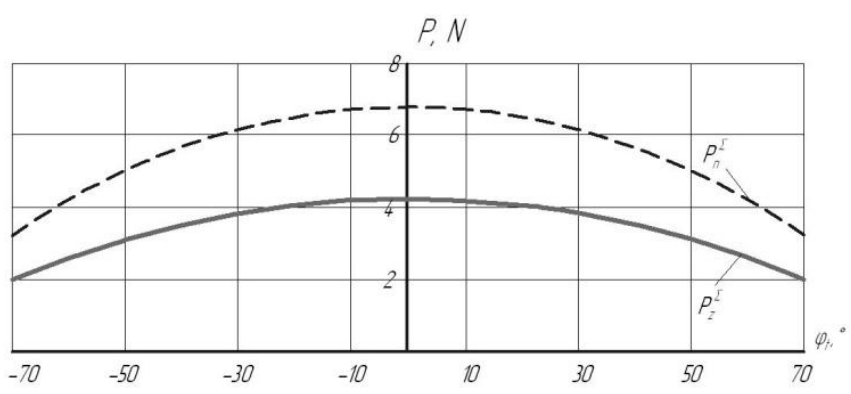

Fig. 14. Distribution of specific grinding force on the arc of the profile of the grinding wheel for the traditional method of processing

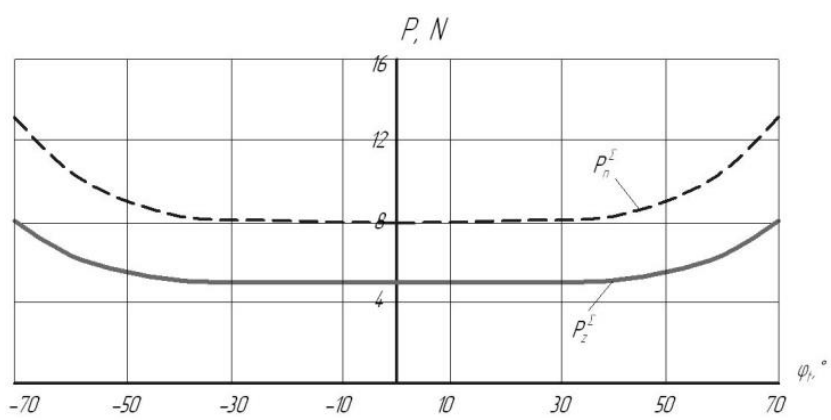

Fig. 15. Distribution of specific grinding force in an profile arc of wheel

Distribution of specific grinding performance, which is described by the inner integral of expression (15) in an arc chute for a traditional processing (curve 1) and the new method (curve 2) are shown in Fig. 12.

As, each cutting edge deforms a metal, the thickness of the shear layer of one of the cutting edge increases gradually from zero to a maximum value at the exit point of the wheel from machining allowance and can be determined from equation (11). The distribution of the shear layer thickness of one cutting edge on the output line of the grinding wheel of the machining allowance for grinding the two methods are shown in Fig. 13.

The specific grinding force i.e. a force that falls on the unit 
of the wheel height is determined by integrating the forces of microcutting $(9,10)$ along the contact patch of the entrance point to the exit point of the range of machining allowance. The distribution of normal $P_{n}$ and tangential $P_{z}$ cutting forces along the arc profile of the grinding wheel, for traditional and new methods are shown in Figs. 14 and 15, respectively.

\section{CONCLUSIONS}

For the first time it has been proposed a method of determining the thickness of a cutting layer of one of the cutting edge, from the condition that the volume of material that is brought and is cut in each local point of contact spots. The proposed method takes into account the compliance of the processing system and the discontinuity of the abrasive surface of the tool.

According to calculations performance of grinding with crossed axes tool and workpiece is higher by reducing the value of infeed, which in this way is equal to the machining allowances. With this method of grinding allowance is cut by equidistant curves, which provides a constant cutting depth. In the traditional method of processing the cutting depth decreases from the center to the edges of the trough (Fig. 11).

The specific grinding performance remains substantially constant within a central angle of $100^{\circ}$, whereas in the traditional method it decreases from the center to the edges of the trough (Fig.12). This provides more uniform volume tool wear generatrix length of a profile.

Processing with crossed axes tool and parts with a profile in the form of a circular arc is especially effective in processing of precise parts, such as rings of ball screw with a central angle of up to $100^{\circ}$.

It is also possible to use a new method for grinding rollers for cold rolling of tubes in which the profile of the central angle exceeds $100^{\circ}$. From the Fig. 13 the thickness a cutting layer of one cutting edge remains virtually constant, which ensures consistent load on the grain. The increase in the specific performance on the edges of the trough is explained by the increase of contact area along the axis $\theta_{t}$ (Fig. 10).

\section{REFERENCES}

1. BrinksmeireE. (2006),Advances in modeling and simulation of grinding processes,Annals of the CIRP, 55,667-696.

2. ChaeJ., ParkS.S., Freiheit T. (2006),Investigation of micro-cutting operations. International Journal of Machine Tools \& Manufacture, 46,313-332.

3. Chang, H.-C., Junz Wang, J.-J.(2008),A stochastic grinding force model considering random grit distribution, International Journal of Machine Tools \& Manufacture, 48, 1335-1344.

4. Chee Keong Ng , Shreyes N. Melkote, M. Rahman, A. Senthil Kumar.(2006), Experimental study of micro- and nano-scale cutting of aluminum 7075-T6,International Journal of Machine Tools \& Manufacture, 46, 929-936.

5. Chen M., LiX., Yang, P. (2007),Study on surface grindinghardening of SKD11 hardened steel,International Journal of Manufacturing Technology and Management, 12, 236-245.

6. HeckerRogelio L. (2007), Grinding force and power modeling based on chip thickness analysis,International Journal of Advanced Manufacturing Technology, 33, 449-459.

7. HeinzelC., BleilN. (2007), The use of the size effect in grinding for work-hardening. Annals of the CIRP, 56, 327-330.

8. Kalchenko V.V., YeroshenkoA.M. (2009),Determination of the efficiency of grinding with crossed axes tool and workpiece with a circular profile, Journal of The National Technical University 'Kharkiv Polytechnic Institute', technologies.- Kharkiv, 1, 52-56.

9. Kalchenko V.I.,KalchenkoV.V.,Pohiba N.N. (2012),Determination of cutting forces in the process of deep grinding surfaces of rotation by oriented elbor wheel, Journal "Super hard materials, 2, 58-73.

10. Lan Yan, Xuekun Li, Feng Jiang, Zhixiong Zhou, Yiming (Kevin) Rong.(2009), Research on microscopic grain-workpiece interaction in grinding, Part 2 - Factorial Analysis. Advanced Materials Research, 76-78, 15-20.

11. MalkinS., GuoC. (2008), Grinding technology - Theory and applications of machining with abrasives, New York: Industrial Press.

12. Nguyen,T. A., Butler, D. L. (2005),Simulation of precision grinding process, part 1: generation of the grinding wheel surface, International Journal of Machine Tools \& Manufacture, $45,1321-1328$

13. Oliveira, J.F.G. (2009),Industrial challenges in grinding,Annals of the CIRP,1-18.

14. Stepien P. (2007), Grinding forces in regular surface texture generation, International Journal of Machine Tools \& Manufacture, 47, 2098-2110.

15. Yeroshenko A.M. (2008), Theoretical and experimental studies of the grinding process with crossed axes tool and the part of the profile curves in a circle, Journal of Chernihiv State Technological University, 35,5-24.

16. Yeroshenko A.M. (2009),Increase of efficiency of polishing with the crossing axes ofinstrument and detail with a type as the arc of circumference, The thesis for a scientific degree in specialty machining processes, machines and tool, National Technical University. 